\title{
Emulsion Stability: Delineation of Different Particle Loss Mechanisms
}

\author{
S. R. REDDY ${ }^{1}$ AND H. S. FOGLER \\ Department of Chemical Engineering, The University of Michigan, Ann Arbor, Michigan 48109
}

Received December 21, 1979; accepted March 10, 1980

\begin{abstract}
The kinetics of the emulsion-breaking process are governed by three different particle loss mechanisms: Brownian flocculation, sedimentation flocculation, and creaming. Various particle loss mechanisms have been delineated and the relative importance of Brownian flocculation and sedimentation flocculation compared. A general map presenting regimes of dominant particle loss mechanisms has been developed from which one can determine the dominant particle loss mechanism for a given particle size and density difference. In addition, the relative importance of flocculation and creaming has been delineated for a monodispersed system. The effects of particle size, surface potential, concentration, density difference, temperature, and ionic strength on the relative importance of flocculation and creaming have been analyzed.
\end{abstract}

\section{INTRODUCTION}

An emulsion is a thermodynamically unstable system and possesses a natural tendency to break. By dispersing one phase into the other, the interface between them is greatly increased, as is the free energy of the interface which is proportional to the interface area. The emulsion, therefore, possesses a natural tendency to separate into two phases.

The kinetics of the emulsion-breaking process are governed by three different particle loss mechanisms: Brownian flocculation, sedimentation flocculation, and creaming. Particles under Brownian motion collide and then coalesce to form larger particles. At the same time, particles are creaming out due to the difference in the densities of the particles and continuous medium. Sedimentation flocculation is due to differential creaming rates of particles of different sizes. The larger particles cream out at a faster rate than the smaller ones; therefore, larger particles collide with slowermoving smaller particles as they cream out.

\footnotetext{
${ }^{1}$ Present address: General Motors Research Laboratories, Fuels and Lubricants Department, Warren, Mich. 48090.
}

In most common emulsions, all the particle loss processes take place simultaneously. A general dynamic equation (GDE) which includes all three processes was derived and solved numerically (1). While the numerical solution to the GDE does accurately predict changes in the particle size and size distribution, it does not delineate the various particle loss processes. It can be explained qualitatively that flocculation will be significant for smaller particles and creaming will be significant for larger particles. However, it is important to develop quantitative relationships to predict the state of an emulsion, i.e., whether emulsion particles are creaming or flocculating, and if both are occurring, which is predominant. In addition, the importance of flocculation resulting from sedimentation will be determined. The object here is to develop maps with domains representing the relative importance of the various particle loss processes as a function of key emulsion properties.

\section{BROWNIAN FLOCCULATION VS SEDIMENTATION FLOCCULATION}

For a given emulsion, the Brownian collision frequency function, $B_{i j}$, and sedimentation collision frequency function, $G_{i j}$, be- 
tween particles of radius $a_{i}$ and $a_{j}$ are given by the expressions $(1-3)$

$$
\begin{aligned}
B_{i j} & =\frac{2 k T}{3 \mu W_{i j}}\left(\frac{1}{a_{i}}+\frac{1}{a_{j}}\right)\left(a_{i}+a_{j}\right), \\
G_{i j} & =\frac{8 \pi \Delta \rho g}{9 \mu W_{i j}}\left(a_{i}^{2}-a_{j}^{2}\right)\left(a_{i}+a_{j}\right)^{2},
\end{aligned}
$$

where

$$
\begin{aligned}
k= & \text { the Boltzmann constant } \\
T= & \text { temperature } \\
\mu= & \text { viscosity } \\
W_{i j}= & \text { the electrostatic stability factor, } \\
\Delta \rho= & \text { the density difference between dis- } \\
& \text { persed phase and continuous phase, } \\
& \text { and } \\
g= & \text { the gravitational constant. }
\end{aligned}
$$

The underlying assumptions in arriving at the equation for the sedimentation collision frequency are that the particle paths are straight vertical lines, the collision efficiency is unity, and the stability factor is identical to that of the Brownian collision frequency. In a related study where the particle size distribution was monitored as a function of time and position, a comparison of theory and experiment shows these assumptions are reasonable (1).

For a given emulsion particle concentration, the rate of collisions between the $i$ th class and the $j$ th class due to Brownian motion and gravitational motion are proportional to $B_{i j}$ and $G_{i j}$, respectively. Therefore, the ratio of Brownian flocculation to sedimentation flocculation is given by

$$
\frac{G_{i j}}{B_{i j}}=\frac{4 \pi \Delta \rho g}{3 k T} a_{i} a_{j}\left(a_{i}^{2}-a_{j}^{2}\right) .
$$

Let $a_{i} / a_{j}=R_{i j}$. Then,

$$
\frac{G_{i j}}{B_{i j}}=\frac{4 \pi \Delta \rho g}{3 k T} a_{i}^{4} R_{i j}\left(1-R_{i j}^{2}\right) .
$$

One observes that this ratio goes through a maximum between $R_{i j}=0$ and $R_{i j}=1$. Differentiating to find the maximum,

$\frac{d\left(G_{i j} / B_{i j}\right)}{d R_{i j}}=\frac{4 \pi \Delta \rho g}{3 k T} a_{i}^{4}\left(1-3 R_{i j}^{2}\right)=0$.
Solving for $R_{i j}$,

$$
R_{i j}=a_{i} / a_{j}=1 / 3^{1 / 2}=0.577 .
$$

In other words, if the particle size distribution were bimodal with the particles of one size being approximately one-half the size of the particles of other size, the ratio of sedimentation flocculation to Brownian flocculation is a maximum.

The ratio $G_{i j} / B_{i j}$ passes through 0 and 1 for a given $a$ and has a maximum value at $R_{i j}=1 / 3^{1 / 2}$. The value of the ratio at the maximum is

$$
\begin{aligned}
\left(\frac{G_{i j}}{B_{i j}}\right)_{\max } & =\frac{4 \pi \Delta \rho g a_{i}^{4}}{3 k T}(1 / 3)^{1 / 2}(1-1 / 3) \\
& =\frac{8 \pi \Delta \rho g}{9(3)^{1 / 2} k T} a_{i}^{4} .
\end{aligned}
$$

We can now determine the particle radii, $\hat{a}_{i}$, for which the rate of sedimentation flocculation will equal the rate of Brownian flocculation:

$$
\left(\frac{G_{i j}}{B_{i j}}\right)_{\max }=1=\frac{8 \pi \Delta \rho g}{9(3)^{1 / 2} k T} \hat{a}_{i}^{4}
$$

or

$$
\left(\hat{a}_{i}\right)=\left(\frac{9(3)^{1 / 2} k T}{8 \pi \Delta \rho g}\right)^{1 / 4} .
$$

For water in paraffin oil, hexatriacontane $\left(\mathrm{C}_{36} \mathrm{H}_{74}\right)$, in water emulsion at $80^{\circ} \mathrm{C}$,

$$
\begin{aligned}
\hat{a}_{i} & =\left(\frac{9(3)^{1 / 2} k T}{8 \pi \Delta \rho g}\right)^{1 / 4} \\
& =\left(\frac{9(3)^{1 / 2} \times 1.3805 \times 10^{-16} \times 353}{8 \pi \times 0.22 \times 980}\right)^{1 / 4}
\end{aligned}
$$

and

$$
\begin{aligned}
& \hat{a}_{i}=1.0881 \times 10^{-4} \mathrm{~cm}, \\
& \hat{a}_{i}=1 \mu \mathrm{m} .
\end{aligned}
$$

The rate of Brownian flocculation between particles with radii 1 and $0.58 \mu \mathrm{m}$ is equal to the rate of sedimentation flocculation between the same particles.

For a paraffin oil-in-water emulsion, the ratio $\left(G_{i j} / B_{i j}\right)$ is plotted as a function of $R_{i j}$ for various values of $D_{i}$ as a parameter in 
Fig. 1. All curves pass through 0 and 1 . The maximum always occurs at $R_{i j}=(1 / 3)^{1 / 2}$.

From these plots, one can estimate the relative importance of Brownian flocculation and sedimentation flocculation. For example, if the emulsion particles are about $1 \mu \mathrm{m}$ in diameter, the Brownian flocculation can be about 15 times greater than the sedimentation flocculation. If the particles are about $4 \mu \mathrm{m}$ in diameter, the sedimentation flocculation can be 10 times greater than the Brownian flocculation. For particles of about $2 \mu \mathrm{m}$ in diameter interacting with particles $1 \mu \mathrm{m}$ in diameter, the Brownian flocculation and sedimentation flocculation will be about the same. Plots of this type provide very useful qualitative information on the stability of an emulsion. Similar plots can be drawn for different values of $\Delta \rho$ and $T$.

\section{FLOCCULATION VS CREAMING}

Emulsion particles of a particular size disappear by any of three processes: Brownian flocculation, sedimentation flocculation, and creaming. Brownian flocculation is due to random thermal motion of particles, creaming is due to gravitational force on the particles, and sedimentation flocculation is due to differential creaming velocities of different size particles. All the loss processes are due to thermal and gravitational forces. If gravitational forces are negligible, the particles will disappear only by Brownian flocculation. Generally, if the particles are very small or if the density of the particles is nearly equal to the density of the medium, the gravitational forces are negligible. Thermal forces will be negligible on larger particles.

Regimes on these various limiting cases can be delineated quantitatively by manipulating the equations for creaming velocity and Brownian and gravitational collision frequency functions. Creaming or settling velocity, $U$, of a particle of radius $a$ is given by the equation

$$
U_{i}=\frac{2 a_{i}^{2}|\Delta \rho| g}{9 \mu},
$$

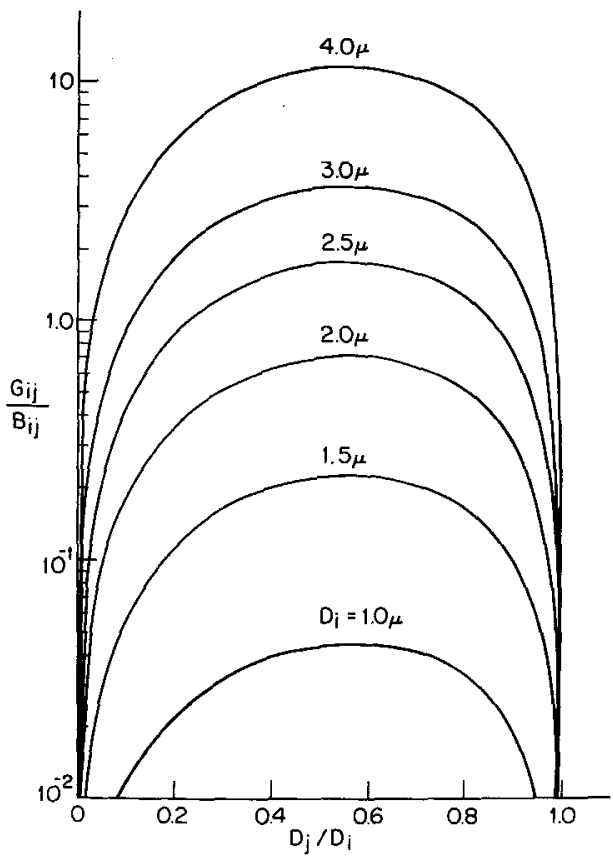

FIG. 1. The importance of sedimentation flocculation and Brownian flocculation.

where

$\Delta \rho=$ the difference between the density of particles and medium,

$g=$ the gravitational constant, and

$\mu=$ the viscosity of the continuous medium.

For a given fluid, the creaming velocity is a strong function of particle size. For very small particles, the creaming velocity will be negligible compared to the thermal motion. A generally accepted rule (3) is that the gravitational forces on the particle can be neglected for creaming velocities of 1 $\mathrm{mm} /$ day or less.

$$
U_{i}=\frac{2 a_{i}^{2}|\Delta \rho| g}{9 \mu}<1 \mathrm{~mm} / \mathrm{day}
$$

or

where

$$
a_{\mathrm{c}}<\left(\frac{9 \mu \times 1.16 \times 10^{-6}}{2|\Delta \rho| g}\right),
$$

$$
\begin{aligned}
\mu & =\text { poise }, \\
\Delta \rho & =\mathrm{g} / \mathrm{cm}^{3}, \text { and } \\
g & =980 \mathrm{~cm} / \mathrm{sec}^{2} .
\end{aligned}
$$




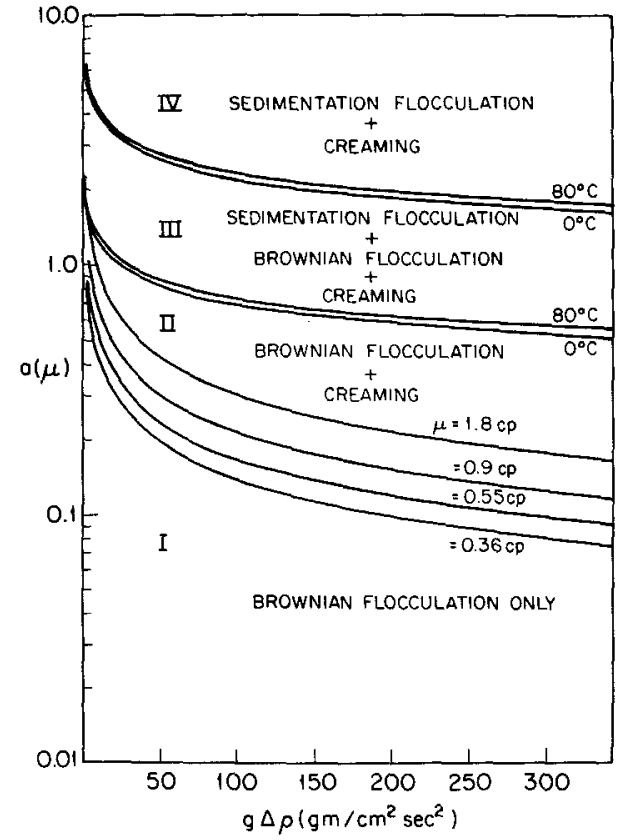

FIG. 2. Regimes of dominant particle loss mechanisms plotted as $a$ vs $g \Delta \rho$.

If particles are smaller than $a_{c}$, they disappear only by Brownian flocculation. If they are larger than $a_{\mathrm{c}}$, they could disappear by any of the three particle loss mechanisms.

From Eq. [6],

$$
\left(\frac{G_{i j}}{B_{i j}}\right)_{R_{i j}=(1 / 3)^{1 / 2}}=\frac{8 \pi \Delta \rho g}{9(3)^{1 / 2} k T} a_{i}^{4} .
$$

Brownian flocculation will be negligible for large values of $G_{i j} / B_{i j}$ and sedimentation flocculation will be negligible for small values of $G_{i j} / B_{i j}$. Therefore, Eq. [6] can be used to define quantitatively the regions where only one of the mechanisms will be predominant. Let us define Brownian flocculation as predominant if the ratio is less than 0.1 and sedimentation flocculation as predominant if it is greater than 10.

$$
\begin{array}{cc}
\frac{8 \pi \Delta \rho g}{9(3)^{1 / 2} k T} a_{i}^{4} \leq 0.1 & \begin{array}{l}
\text { (Brownian } \\
\text { flocculation), }
\end{array} \\
\frac{8 \pi \Delta \rho g}{9(3)^{1 / 2} k T} a_{i}^{4} \geq 10 & \begin{array}{c}
\text { (sedimentation } \\
\text { flocculation) }
\end{array}
\end{array}
$$

The key parameters in Eqs. [10], [11], and [12] are particle radius $a_{i}$ and density difference $\Delta \rho$. Therefore, $a$ and $\Delta \rho$ can be used to map the various domains for the particle loss mechanisms.

In a more general way, plots of $a$ vs $g \Delta \rho$ were prepared by using Eqs. [10], [11], and [12]. The domains are shown in Fig. 2. Equation [10] was used to obtain the upper boundary of region I, and Eqs. [11] and [12] were used to obtain the lower boundary and the upper boundary of region III, respectively. The figure can be used for any system provided that the temperature and viscosity of the continuous medium correspond to the values shown in the figure. It can also be used for centrifugal settling instead of gravitational settling. For centrifugal settling, $g$ is to be replaced by $w^{2} X$, where $w$ is the rate of rotation and $X$ is the distance from the center.

Various regions in Fig. 2 represent the possible dominant particle loss process. For example, in region I creaming and sedimentation will be negligible and particles may disappear only by Brownian flocculation. Brownian flocculation in region I will depend on the surface potential of the particles. If the particles carry a large surface potential, even the Brownian flocculation will be negligible. Brownian flocculation will also be negligible if the emulsion is very dilute. If a particular emulsion falls in region $I$, one can neglect creaming and sedimentation flocculation terms in the general dynamic equation.

If an emulsion's properties are in region II, sedimentation flocculation will be negligible when compared to Brownian flocculation. Creaming is bound to occur and the rate of Brownian flocculation will depend on surface potential and concentration of particles. Similarly, in region IV Brownian flocculation will be negligible. Creaming is bound to occur and the rate of sedimentation flocculation depends on surface potential, particle concentration, and polydispersity of the emulsion. 
Figure 2 is useful in determining the state of an emulsion, i.e., whether the emulsion particles are creaming or flocculating. If they are flocculating, what type of flocculation is occurring? One can then solve the pertaining general dynamic equation to predict the behavior of the emulsion. In the case of a polydispersed system, the plot tells the type of mechanism acting on a particular size particle.

\section{EFFECT OF PHYSICAL AND ELECTROSTATIC PROPERTIES ON RELATIVE RATES OF FLOCCULATION AND CREAMING}

In general, a high surface potential keeps the particles stable against flocculation while a small particle size and negligible density difference between particles and continuous medium keep the particles stable against creaming. The relative importance of the two processes has not been extensively delineated for given physical and electrostatic properties of the emulsion. From previous sections, one can determine which one of the processes will be negligible for a given particle size and density difference. In this section, the relative importance of the processes for a given particle size, density difference, ionic strength, surface potential, and particle concentration will be discussed. The information is derived analytically by assuming a monodispersed or very nearly monodispersed system.

For a monodispersed system the general dynamic equation reduces to

$$
\frac{\partial N}{\partial t}=-U \frac{\partial N}{\partial y}-B N^{2},
$$

where

$N=$ the concentration of monodispersed particles,

$U=$ the creaming velocity,

$B=$ the Brownian collision frequency function,

$y=$ the distance in the direction of gravity, and

$t=$ time.
The creaming term can be further approximated (2) as

$$
\frac{\partial N}{\partial y}=\frac{N}{h},
$$

where $h$ is the height of the column. This approximation is reasoned as follows: the product of the particle concentration and creaming velocity, $N U$, gives the rate of the total number of particles creaming from the system per unit area, and $U N / h$ is the rate of loss of particles per unit volume by creaming. The general equation reduces to

$$
\frac{d N}{d t}=-U \frac{N}{h}-B N^{2} .
$$

The ratio of the rate of flocculation, $R_{\mathrm{f}}$, to the rate of creaming, $R_{\mathrm{c}}$, is given by

$$
R=\frac{R_{\mathrm{f}}}{R_{\mathrm{c}}}=\frac{B N^{2}}{U(N / h)} .
$$

The expressions for $B$ and $U$ are

$$
\begin{aligned}
B & =\frac{4}{3} \frac{k T}{\mu W}, \\
U & =\frac{2 g a^{2} \Delta \rho}{9 \mu} .
\end{aligned}
$$

Substituting [17] and [18] into [16],

$$
R=\frac{6 k T}{a^{2} W}\left(\frac{h N}{g \Delta \rho}\right) .
$$

An approximate and useful relationship for the stability factor, $W$, for the case when the potential energy of interaction is at least five times greater than the thermal energy, $k T$, is given by $(4,5)$

$$
W=\frac{2 \pi^{1 / 2} \exp \left(V_{T_{\max }} / k T\right)}{\left(u_{\max }+2\right)^{2}\left(-V_{T_{\max }}^{\prime \prime} / 2 k T\right)^{1 / 2}},
$$

where

$$
-\frac{V_{T_{\text {max }}}^{\prime \prime}}{2 k T}=\frac{A a^{3} K^{3}}{24 k T}
$$

and

$$
\begin{aligned}
V_{T \max } & =\frac{a}{2}\left[\epsilon \Psi_{0}^{2} \ln \left(1+e^{-1}\right) .\right. \\
& \left.-\frac{A}{6 K^{-1}}\left(\frac{10^{-5}}{10^{-5}+11.116 K^{-1}}\right)\right]
\end{aligned}
$$




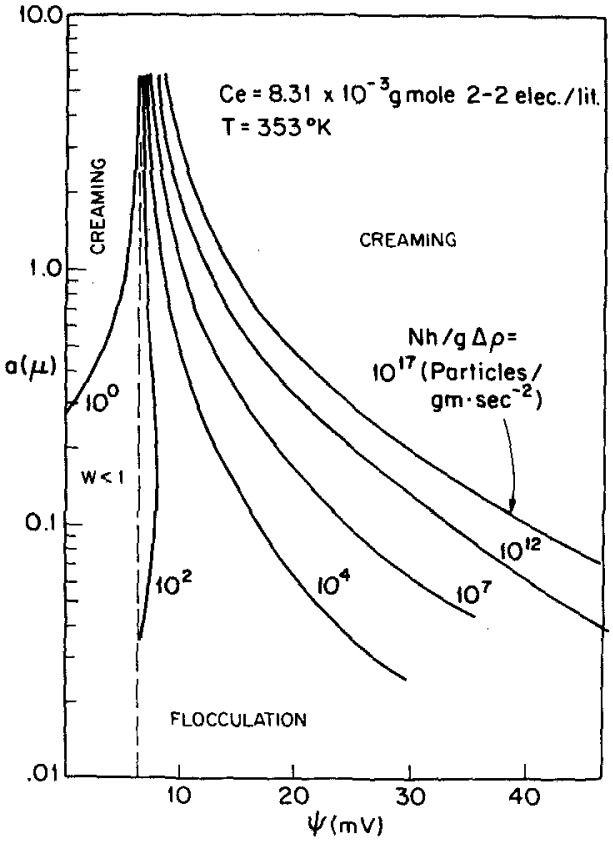

FIg. 3. Regimes of dominant particle loss mechanism plotted as $a$ vs $\psi_{0}$ and $N h / g \Delta \rho$ as parameter.

and when the particle radius is greater than the double-layer thickness, $\left(u_{\max }+2\right) \simeq 2$.

With these approximations Eq. [20] reduces to

$$
\begin{aligned}
W= & \frac{\pi^{1 / 2}}{2}\left[\left(\frac{24 k T}{A a^{3} K^{3}}\right)\right]^{1 / 2} \\
& \times \exp \left[\frac { a } { 2 k T } \left(\epsilon \Psi_{0}^{2} \ln \left(1+e^{-1}\right)\right.\right. \\
& \left.-\frac{A}{6 K^{-1}}\left(\frac{10^{-5}}{10^{-5}+11.116 K^{-1}}\right)\right],
\end{aligned}
$$

where

$$
K=\left(\frac{24 k T}{4 \pi e^{2} \sum n_{i} z_{i}^{2}}\right)^{1 / 2}
$$

where

$k=$ the Boltzmann constant,

$K=$ the reciprocal of the double-layer thickness,

$T=$ temperature,

$a=$ the particle radius,

$H_{\max }=$ the shortest interparticle distance when $V_{T}$ is maximum,

$$
\begin{aligned}
u_{\max } & =H_{\max } / a \\
\epsilon & =\text { the dielectric constant, } \\
\Psi_{0}= & \text { the surface potential, } \\
A & =\text { the Hamaker constant, } \\
e & =\text { the electronic charge, } \\
n_{\mathrm{i}} & =\text { the concentration of ions, } \mathrm{i}, \text { per } \\
& \text { cubic centimeter, and } \\
z_{\mathrm{i}} & =\text { the valence of ions, } \mathrm{i} .
\end{aligned}
$$

Substituting Eq. [23] into [19],

$$
\begin{aligned}
R= & 6 k T\left(\frac{h N}{\Delta \rho g}\right)\left(\frac{6 \pi k T a}{A K^{3}}\right)^{-1 / 2} \\
& \times \exp \left(-\frac{a}{2 k T}\left[0.3133 \epsilon \Psi_{0}^{2}\right.\right. \\
& \left.\left.-\frac{A K}{6}\left(\frac{10^{-5}}{10^{-5}+11.116 K^{-1}}\right)\right]\right) .
\end{aligned}
$$

This equation can best be solved for $\Psi_{0}$ :

$$
\begin{aligned}
\Psi_{0}= & \frac{1}{0.3133 \epsilon}\left[\frac{A K}{6}\left(\frac{10^{-5}}{10^{-5}+11.116 K^{-1}}\right)\right. \\
+\frac{2 k T}{a} \times & \ln \left[6 k T\left(\frac{h N}{\Delta \rho g R}\right)\right. \\
& \left.\left.\left.\times\left(\frac{6 \pi k T a}{A K^{3}}\right)^{-1 / 2}\right]\right]\right)^{1 / 2} \cdot
\end{aligned}
$$

Equation [25] can be used to develop the domains of the importance of various particle loss mechanisms. By equating the rate of flocculation to the rate of creaming, $R=1$, one can determine the relationship between $\psi$ and the particle radius for all other given properties. In Fig. 3 the surface potential is plotted as a function of the particle radius for a given ionic strength and temperature for various values of the parameter $h N / \Delta \rho g$. If $h N / g \Delta \rho$ and the particle radius are known, one can determine from Fig. 3 whether the creaming is important and the value of $\psi$ necessary to keep the particles stable against flocculation.

If one knows all the properties of the emulsion, it is possible to determine the ratio of the rate of flocculation to the rate of creaming, $R$. This can be achieved by treating the quantity $(N h / g \Delta \rho)$ in Fig. 3 as $(N h / g \Delta \rho R)$. If the particle radius, $a$, surface potential, 
$\psi$, particle concentration, and density difference are known, one can find the ratio of the rate of flocculation to the rate of creaming, $R$. For example, for $\psi=20 \mathrm{mV}$ and $a$ $=0.5 \mu \mathrm{m}$ one finds from Fig. 3

$$
\frac{N h}{g \Delta \rho R}=10^{17} \text {. }
$$

For particle concentration of $10^{13}$ particles/ $\mathrm{cm}^{3}$, height of $9.8 \mathrm{~cm}$, and a $\Delta \rho$ of $0.1 \mathrm{~g} / \mathrm{cm}^{3}$, the

$$
\frac{N h}{g \Delta \rho}=10^{12}
$$

or $R=10^{-5}$. For this particular system, the rate of creaming will be $10^{5}$ times greater than the rate of flocculation. From Fig. 3 one also observes, for a particle radius of 0.325 $\mu \mathrm{m}$ instead of $0.5 \mu \mathrm{m}$, the rate of creaming and flocculation will be equal. For the same system, the rate of flocculation will be equal to the rate of creaming if the surface potential is $16 \mathrm{mV}$ instead of $20 \mathrm{mV}$. In other words, if the system falls below the line corresponding to its $N h / g \Delta \rho$, the particles will be mostly flocculating; above the line they will be mostly creaming. For this system, if the particle radius is less than $0.4 \mu \mathrm{m}$ and $\psi$ is less than $15 \mathrm{mV}$, virtually all the loss of particles from the emulsion will result from flocculation. If the particle radius is greater than $0.6 \mu \mathrm{m}$ and $\psi$ is greater than $23 \mathrm{mV}$, particles will be lost by creaming. If the particle radius and surface potential are in between these ranges, particles will be lost by simultaneous flocculation and creaming. Figure 3 with various domains is very useful in determining the state of an emulsion and the effect of various parameters on the stability of emulsions.

The effect of electrolyte concentration is shown in Fig. 4, where surface potential is plotted as a function of particle radius for a given $N h / g \Delta \rho$ and temperature. From this figure, one can also determine the electrolyte concentration at which flocculation and creaming become equal, or for a given concentration of electrolyte, the dominating

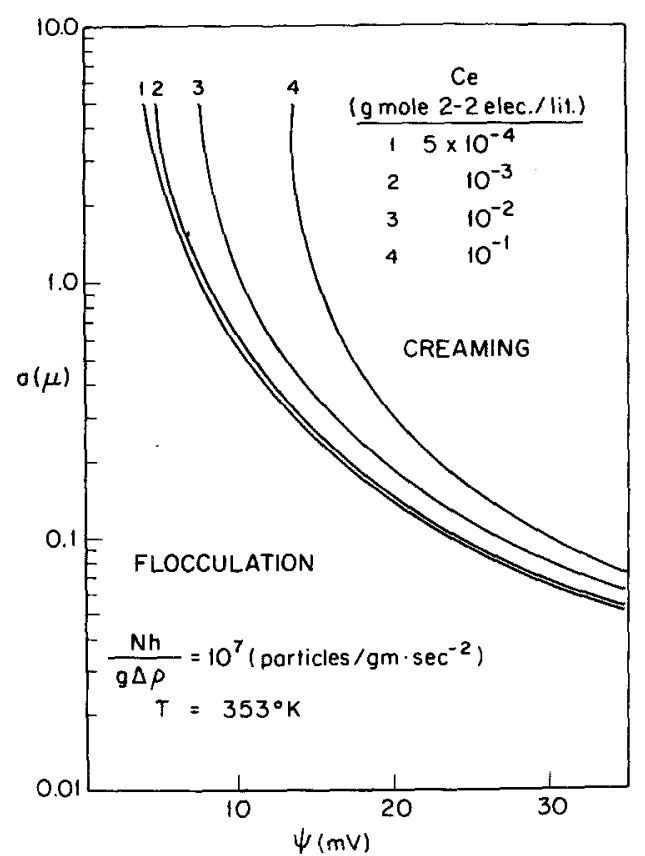

FIG. 4. Regimes of dominant particle loss mechanism plotted as $a$ vs $\psi_{0}$ and $C_{\mathrm{e}}$ as parameter.

particle loss mechanism. For example, if $C_{\mathrm{e}}$ $=10^{-2}$ gmole/liter, the particle radius is greater than $0.5 \mu \mathrm{m}$, and $\psi$ is greater than $15 \mathrm{mV}$, the particles will be mostly creaming; for the same electrolyte concentration, if the particle radius is less than $0.4 \mu \mathrm{m}$ and $\psi$ is less than $10 \mathrm{mV}$, they will be mostly flocculating and in between these values particles will be lost by flocculation and creaming simultaneously.

The effect of temperature is shown in Fig. 5. For most practical oil/water emulsions the increase in temperature increases the rate of flocculation and the rate of creaming without much change in the ratio of these rates. However, large increases in temperature (feasible in colloid sols or special emulsions under pressure) will increase the ratio of the rate of flocculation to the rate of creaming, as seen in Fig. 5.

In the case of unstable emulsions such as water-in-oil emulsions without any stabilizer, the stability factor, $W$, can be equated to unity. Equation [19] reduces to 


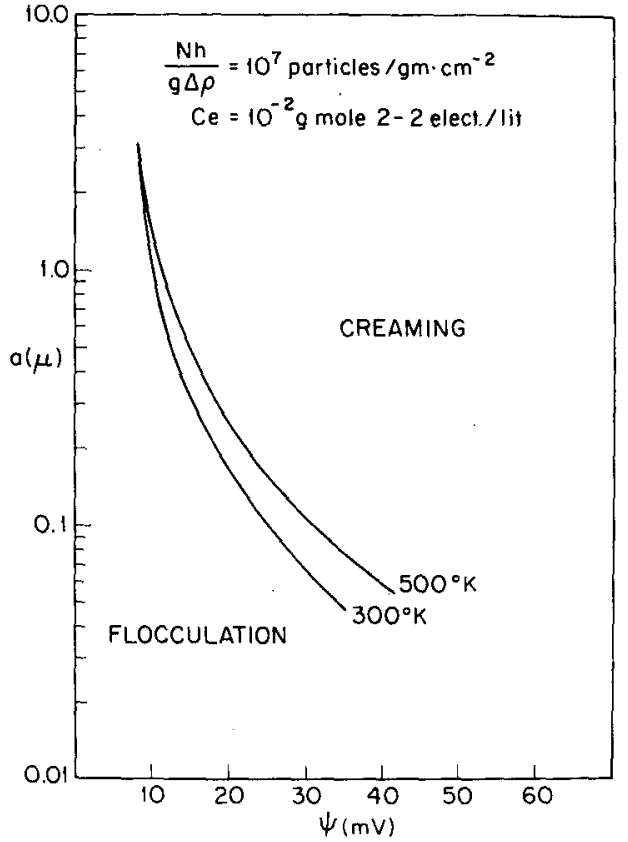

FIg. 5. Regimes of dominant particle loss mechanism plotted as $a$ vs $\psi_{0}$ and $T$ as parameter.

$$
R=\frac{6 k T}{a^{2}}\left(\frac{h N}{g \Delta \rho}\right) .
$$

Equation [26] can be written in terms of the volume fraction of the dispersed phase, $\phi$ :

$$
R=\frac{9 k T}{2 \pi} \frac{1}{a^{5}}\left(\frac{h \phi}{g \Delta \rho}\right) .
$$

Solving Eq. [27] for the particle radius, $a$,

$$
a=\left[\frac{9 k T}{2 \pi}\left(\frac{h \phi}{R g \Delta \rho}\right)\right]^{1 / 5} .
$$

Equation [28] was used to map the domains of the dominant particle loss mechanism for water-in-oil emulsions, as shown in Fig. 6. The ratio of the rate of flocculation to the rate of creaming can be determined in the same manner as explained for Fig. 3 . In the case of water-in-oil emulsions, flocculation is dominant unless the particles are very large or the concentration is very low in which case creaming becomes dominant.

The total rate of loss of particles due to flocculation and creaming can be determined from Figs. 3 to 6 . One can determine the ratio of rates, $R$, from Figs. 3 to 6 for given properties of the emulsion. The rate of creaming can easily be calculated from

$$
R_{\mathrm{c}}=\frac{2 g a^{2} \Delta \rho}{9 \mu} \frac{N}{h} \text {. }
$$

Knowing the rate of creaming, $R_{\mathrm{c}}$, and the ratio of the rate of flocculation to the rate of creaming, $R$, and the total rate of loss of particles from the unit volume, one can compute the rate of flocculation. For example, consider the emulsion with the properties shown in Fig. 3 and $a=0.5 \mu \mathrm{m}, \psi=20 \mathrm{mV}$, $h=10 \mathrm{~cm}, \Delta \rho=0.2 \mathrm{~g} / \mathrm{cm}^{3}, \mu=0.37 \mathrm{cP}$, and $N h / g \Delta \rho=10^{17}$. From Fig. 3, $R=1$ and $R_{\mathrm{c}}$ is given by

$$
\begin{aligned}
R_{\mathrm{c}} & =\frac{2 \times 980 \times\left(0.5 \times 10^{-4}\right)^{2} \times 2}{9 \times 0.37 \times 10^{-2}} \frac{1.96 \times 10^{19}}{10} \\
& =5.768 \times 10^{13} \mathrm{particles} / \mathrm{cm}^{3} \cdot \mathrm{sec} .
\end{aligned}
$$

Therefore, the loss of monodispersed particles per unit volume per unit time due to flocculation and creaming is $1.1436 \times 10^{14}$. Since the emulsion under consideration is monodispersed, the loss of particles by flocculation will result in a gain of particles of larger size.

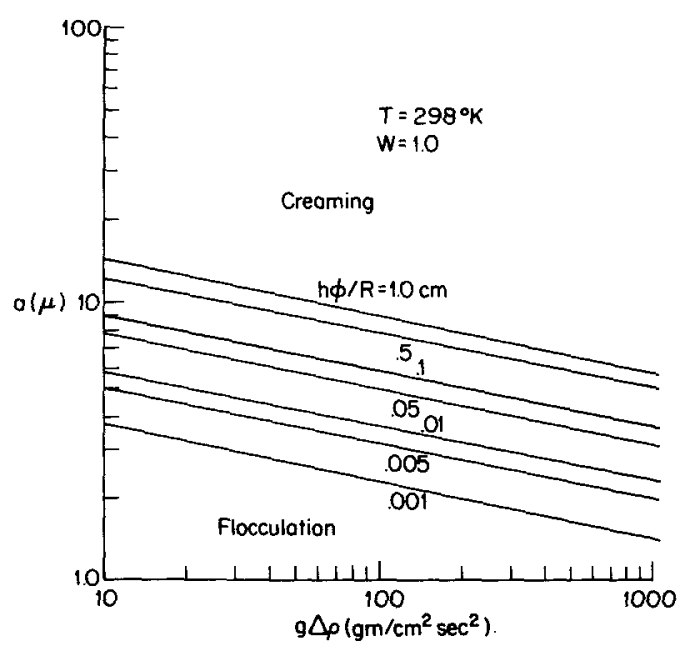

FIG. 6. Regimes of dominant particle loss mechanism plotted as $a$ vs $g \Delta \rho$ and $h \phi / R$ as parameter for water-in-oil emulsions. 
The maps of various types with regimes presenting the dominant particle loss mechanisms are very useful in predicting the semiquantitative information about the stability of emulsions. The maps can also be used for preparing the emulsions of desired stability. However, for accurate information on the stability of emulsions, one needs to solve the general dynamic equation (1).

\section{SUMMARY}

The relative importance of Brownian flocculation and sedimentation flocculation was delineated. If the density difference between the particles and the continuous medium is about $0.22 \mathrm{~g} / \mathrm{cm}^{3}$, Brownian flocculation is significant for particles of diameter smaller than $1 \mu \mathrm{m}$ and sedimentation flocculation for particles larger than $2 \mu \mathrm{m}$.

A general map for any emulsion, depicting the regimes of dominant particle loss mechanism, was developed. From this map one can determine the qualitative information about the stability of an emulsion for a given particle size and density difference.

The relative importance of Brownian flocculation and creaming was investigated. General maps were prepared showing the effect of various physical and electrochemical properties on the relative rates of flocculation and creaming. It is possible to obtain useful information on the stability of an emulsion before proceeding to complicated mathematical computations.

\section{REFERENCES}

1. Reddy, S. R., "On the Stability of Emulsions," Ph.D. thesis, The University of Michigan, 1980.

2. Friedlander, S. K., "Smoke, Dust and Haze, Fundamentals of Aerosol Behavior." Wiley, New York, 1977.

3. Druyt, K., "Colloid Science," Vol. 1, Chap. 7. Elsevier, Amsterdam, 1952.

4. Reerink, H., and Overbeek, J. Th. G., Discuss. Faraday Soc. 18, 74 (1954).

5. Suzuki, A., Ho, N. F. H., and Higuchi, W. I., J. Colloid Interface Sci. 29, 552 (1969). 